\title{
A Comparison of Clinical Surveillance Systems in New York City
}

\author{
Lauren Schreibstein ${ }^{\star 1}$, Remle Newton-Dame1, Katharine H. McVeigh¹, Sharon E. \\ Perlman', Lorna E. Thorpe ${ }^{2}$, Hannah Mandel' ${ }^{1}$ and Michael Buck ${ }^{1}$
}

${ }^{1}$ Primary Care information Project, NYC Dept of Health and Mental Hygiene, Long Island City, NY, USA; ${ }^{2}$ City University of New York, New York, NY, USA

\section{Objective}

To compare two clinical surveillance systems in development in New York City, one built on a distributed query network of electronic health records (EHRs) and the other accessing data from a Health Information Exchange (HIE).

\section{Introduction}

The widespread adoption of Electronic Health Records and the formation of Health Information Exchanges has opened up new possibilities for public health monitoring. Since 2009, The New York City (NYC) Department of Health and Mental Hygiene (DOHMH) has been developing two public health surveillance systems for chronic diseases. The first is the NYC Macroscope, which is built on a distributed query network (the Hub) of 740 New York City ambulatory practices all using proprietary software from one EHR vendor (eClinicalWorks). The second model, Query Health, still in its initial phase, accesses data collected by Healthix, the largest NYC HIE. This study compares these two models for potential disease surveillance and public health application.

\section{Methods}

In the Query Health model, the data has already been collected and standardized by Healthix and is transmitted in HL 7 C 32 standards, one document per patient. Data is available from $100 \%$ of the facilities, but facilities are providing Healthix with varying areas of the record. For NYC Macroscope, queries are developed and executed nightly on each individual practice's EHR. Because queries are being executed against the live practice database, individual practices may not send a response to every query, but all areas of the record are being accessed for each practice that does return data. Poor data documentation at certain practices is a challenge in both systems. Inclusion criteria for Hub data were applied such that annually each provider minimally has a) seen 10 adult patients, b) documented BMI or BP on $>=50 \%$ of patients, c) prescribed a medication to $>=20 \%$ of patients, and d) documented $1+$ ICD- 9 codes for $>=80 \%$ of patients. Individual provider documentation has not yet been assessed for Query Health.

\section{Results}

The Hub had 6 million patients who ever had a visit at one of the 740 participating ambulatory practices, representing approximately $9 \%$ of the estimated over 8,000 NYC practices. This includes nonNYC residents and has not been de-duplicated for patients visiting multiple practices. Ninety-three percent $(n=686)$ of Hub practices returned data for the NYC Macroscope queries. For 2013, 700,000 adult patients with an eligible visit to a primary care provider that met the inclusion criteria were included in the Macroscope.

The participating Query Health HIE had 26 of the 54 (48\%) NYC hospitals, an additional 15 hospitals on Long Island, and 61 non-hospital facilities submitting patient demographic data for approximately 9 million patients with both in-patient and ambulatory visits. Current data coverage is variable with $94 \%$ of facilities submitting encounter data, $80 \%$ submitting diagnosis data, $65 \%$ submitting lab results, 39\% submitting vital signs, and 24\% submitting medications. Practice documentation will also be a factor for Query Health and inclusion criteria will be applied for future surveillance efforts.

\section{Conclusions}

The NYC Macroscope has provided a novel view for chronic disease surveillance on a scale not previously seen for the health of New Yorkers. This model allows more complete access to the medical record, but is limited to participating practices returning data nightly. Unlike the Macroscope, Query Health has access to data for all participating facilities, but not all areas of the patient record are currently available in Query Health. The areas in patient records currently available for Query Health have potential for estimating disease prevalence. As Query Health facilities have limited vital sign and medication data, the Macroscope may be a more complete system for disease treatment and control estimates. As HIEs expand and receive more areas of the patients' record, Query Health has the potential to be a large scale source for public health surveillance. Continued work is needed with HIEs and to support improvement of data completeness and quality, especially for public health use cases, in NYC and beyond.

\section{Keywords}

Chronic disease surveillance; Surveillance system; Health Information Exchange

\section{*Lauren Schreibstein}

E-mail: Ischreibstein@health.nyc.gov 